\title{
Extending Choquet integrals for aggregation of ordinal values
}

\author{
Lluís Godo \\ Institut d'Investigació en Intel-ligència Artificial \\ IIIA - CSIC \\ Campus UAB s/n, 08193 Bellaterra, Spain \\ gododiia.csic.es
}

\author{
Vicenç Torra \\ Institut d'Investigació en Intel-ligència Artificial \\ IIIA- CSIC \\ Campus UAB s/n, 08193 Bellaterra, Spain \\ vtorra@iiia.csic.es
}

\begin{abstract}
In this paper we study the extension of Choquet integrals to ordinal scales. We show that two different integrals can be defined on the basis of two different but equivalent expressions in the numerical case. We prove some properties of the Choquet integrals and show their application to aggregation.
\end{abstract}

Keywords: Aggregation operators, qualitative aggregation, Choquet integral.

\section{Introduction}

Several aggregation operators have been defined up to now in the area of artificial intelligence for decision making. Different methods have been defined to be applied to different type of objects. For example, there exist aggregation operators for numerical values, ordinal preferences, partitions, and so on.

In the numerical setting, probably the best known and more extensively used aggregation operator is the Weighted Mean (WM), characterized in [Aczel, 1984], that computes a linear combination of values according to a set of weights that account for the importance or reliability of the information sources that supply the values. This function behaves well when the information sources are in some sense independent but it does not when there are interactions between them. In such a situation, alternative operators based on the Choquet integral [Choquet, 1968] are much more appropriate (see [Grabisch et al., 1995] for an overview). Choquet integrals also compute linear combinations of values but with respect to a fuzzy measure (a monotonic but possibly non-additive measure). Such a measure defined over subsets of information sources allows the user to express the interactions between the sources so that the final value of the aggregation reduces the effect of having redundant sources. Moreover, Choquet integrals have the property of reducing to a weighted mean when no interaction exists among the sources, therefore being a generalization of the weighted mean. Also, when measures are symmetric - or commutative - (the measure of a set does not depend on the elements of the set but only on the cardinality of the set) it reduces to an Ordered Weighted Averaging (OWA) operator [Yager, 1988].

In [Godo and, Torra, 1999] the authors propose and define an aggregation operator for ordinal values that is the corresponding counterpart of the numerical weighted mean. Already existing procedures to aggregate ordinal information are mainly based on the Sugeno integral [Sugeno, 1974] (e.g. the Weighted Minimum or the Weighted Maximum [Dubois and Prade, 1986]), and they can be seen as generalized medians rather than means, and therefore they lack a notion of average. When a notion of average is required, the usual procedure was to assume a mapping of ordinal values and weights into a numerical scale and perform the aggregation in this scale (e.g., the Linguistic OWA operator [Herrera and Verdegay, 1993]). The Qualitative Weighted Mean (QWM) defined in [Godo and Torra, 1999] behaves like an average without having to necessarily use any numerical interpretation of the values.

However, the same problem that appears when considering the numerical weighted mean under the presence of interacting information sources is present in the case of the qualitative weighted mean. Therefore, there is the need of defining a qualitative counterpart of the numerical Choquet integral. In [Godo and Torra, 1999] the authors already point out 
to a possible version of such an operator based on the QWM. Here, besides of generalizing it to some extent and studying its properties in detail, we also propose an alternative formulation of a qualitative Choquet integral. We show also that we can see this last operator in the general Murofushi and Sugeno's framework of Fuzzy Integrals [Murofushi and Sugeno, 1991].

The structure of the paper is as follows. In Section 2 we establish the basics for the definition of the Choquet integral and review two equivalent expressions to compute it in the quantitative case. In Section 3 we extend one of the expressions to the qualitative case. Some properties of this generalization of the Choquet integral are given in Section 4. Section 5 is devoted to the second generalization. The work finishes with an example in Section 6 and giving some conclusions in Section 7.

\section{Towards a qualitative Choquet integral for aggregation}

Let $\mu: 2^{X} \rightarrow[0,1]$ be a fuzzy measure on a discrete set $\mathrm{X}=\left\{\mathrm{x}_{1}, \ldots, \mathrm{x}_{\mathrm{n}}\right\}$. The Choquet integral of a function $\mathrm{f}: \mathrm{X} \rightarrow[0,1]$ with respect to $\mu$ is defined as

(C) $\int \mathrm{f} d \mu=$

$$
\Sigma_{\mathrm{k}=1, \mathrm{n}} \mathrm{f}\left(\mathrm{x}_{\sigma(\mathrm{k})}\right)\left[\mu\left(\mathrm{A}_{\sigma(\mathrm{k})}\right)-\mu\left(\mathrm{A}_{\sigma(\mathrm{k}+1)}\right)\right]
$$

where $f\left(x_{\sigma(i)}\right)$ indicates that the indices have been permuted so that $0 \leq \mathrm{f}\left(\mathrm{x}_{\sigma(1)}\right) \leq \ldots \leq \mathrm{f}\left(\mathrm{x}_{\sigma(\mathrm{n})}\right) \leq 1$, $A_{\sigma(i)}=\left\{x_{\sigma(i)}, \ldots, x_{\sigma(n)}\right\}$ for $i \leq n$, and $A_{\sigma(n+1)}=\varnothing$. An alternative equivalent expression is

(C) $\int \mathrm{f} d \mu=\Sigma_{\mathrm{k}=1, \mathrm{n}}\left(\mathrm{f}\left(\mathrm{x}_{\sigma(\mathrm{k})}\right)-\mathrm{f}\left(\mathrm{x}_{\sigma(\mathrm{k}-1)}\right)\right) \mu\left(\mathrm{A}_{\sigma(\mathrm{k})}\right)$

where $\mathrm{f}\left(\mathrm{x}_{\sigma(0)}\right)=0$. Expression $(\mathrm{C} 1)$ shows that the Choquet integral is actually a linear combination of the values $f\left(x_{\sigma(k)}\right)$ with a particular set of weights. Actually, it can be proven that the Choquet integral reduces to the weighted mean when the fuzzy measure is additive, i.e. when $\mu(\mathrm{A} \cup \mathrm{B})=\mu(\mathrm{A})+$ $\mu(\mathrm{B})$ if $\mathrm{A} \cap \mathrm{B}=\varnothing$, and reduces to the OWA operator [Yager, 1988] when the fuzzy measure is commutative, i.e. when $\mu(\mathrm{A})=\mu(\mathrm{B})$ for all $\mathrm{A}, \mathrm{B}$ with the same cardinality.

Our final aim is to extend the Choquet integral to be used for aggregation in an ordinal or qualitative framework. By this we mean to aggregate values belonging to a finite ordinal scale $\mathrm{U}=\left\{\mathrm{O}_{\mathrm{U}}=\mathrm{u}_{0}<\mathrm{u}_{1}\right.$ $\left.<\mathrm{u}_{2}<\ldots<\mathrm{u}_{\mathrm{r}-1}<\mathrm{u}_{\mathrm{r}}=1_{\mathrm{U}}\right\}$, values being the result of evaluation of an object with respect to a set of criteria $\mathrm{X}$, and according to a weighting measure for sets of criteria. Such a measure is assumed to be defined as a set function

$$
\mu: 2^{\mathrm{X}} \rightarrow \mathrm{W}
$$

where $(\mathrm{W},<)$ is just a finite scale $\left\{\mathrm{w}_{0}<\mathrm{w}_{1}<\ldots<\right.$ $\left.\mathrm{w}_{\mathrm{n}}\right\}$, and $\mu$ fulfils the usual requirements of a fuzzy measure, i.e.

$$
\begin{aligned}
& -\mu(\varnothing)=w_{0} \\
& -\mu(X)=w_{n}
\end{aligned}
$$

$-\mu$ is monotone, i.e. if $\mathrm{A} \subset \mathrm{B}$ then $\mu(\mathrm{A}) \leq \mu(\mathrm{B})$.

Since $\mathrm{W}$ is intended to be only a qualitative scale, only the ordering is what really matters and thus, for the sake of a simpler notation, we can safely consider $\mathrm{W}$ to be just a set of ordered indices, that is, $\mathrm{W}=\{0,1, \ldots, \mathrm{n}\}$, for some natural $\mathrm{n}$. Moreover, assume we have $\mathrm{k}$ criteria $\mathrm{c}_{1}, \ldots, \mathrm{c}_{\mathrm{k}}$ and the evaluations over $U=\left\{0_{U}<u_{1}<u_{2}<\ldots<u_{1-1}<1_{U}\right\}$ of an object with respect to these criteria are $\mathrm{v}_{1}, \ldots$, $\mathrm{v}_{\mathrm{k}}$. Let $\{\sigma(1), \ldots, \sigma(\mathrm{k})\}$ be a permutation of $\{1, \ldots, \mathrm{k}\}$ such that $\mathrm{v}_{\sigma(1)} \leq \mathrm{v}_{\sigma(2)} \leq \ldots \leq \mathrm{v}_{\sigma(\mathrm{k})}$, and let $\mathrm{A}_{\sigma(\mathrm{r})}=$ $\left\{c_{\sigma(j)} \mid j \geq k\right\}$, therefore $A_{\sigma(r)}=\left\{c_{\sigma(r)}, . . c_{\sigma(n)}\right\}$ when $\mathrm{r} \leq \mathrm{n}$ and $\mathrm{A}_{\sigma(\mathrm{n}+1)}=\varnothing$.

As already mentioned, we want to mimic the Choquet integral to aggregate the ordinal values $v_{1}$, $\ldots, v_{k}$ according to the measure $\mu$. The first question we have to address is where do we start from, either from expression $(\mathrm{C} 1)$ or expression $(\mathrm{C} 2)$, since both are equivalent in the numerical setting. However, as we shall see, this equivalency is easily lost when moving to our qualitative framework. In the next sections we explore both alternatives.

\section{Qualitative Choquet integral of type I}

We want to generalize the expression

(C) $\int \mathrm{f} d \mu=\Sigma_{\mathrm{k}=1, \mathrm{n}} \mathrm{f}\left(\mathrm{x}_{\sigma(\mathrm{k})}\right)\left[\mu\left(\mathrm{A}_{\sigma(\mathrm{k})}\right)-\mu\left(\mathrm{A}_{\sigma(\mathrm{k}+1)}\right)\right]$

in order to apply it in our qualitative framework above introduced. Recall that in such a framework we want to aggregate evaluations of an object w.r.t. a set of criteria, evaluations which are over an ordinal scale $(\mathrm{U},<)$, and according to a weighting measure $\mu: 2^{\mathrm{X}} \rightarrow \mathrm{W}$ for sets of criteria. Here $(\mathrm{W},<)$ is just another finite scale $\left\{\mathrm{w}_{0}<\mathrm{w}_{1}<\ldots<\mathrm{w}_{\mathrm{n}}\right\}$, and 
$\mu$ fulfils the usual requirements of a fuzzy measure (see above).

First, observe that we need a subtraction-like operation to evaluate the difference between pairs of measures $\mu\left(\mathrm{A}_{\sigma(\mathrm{i})}\right)$ and $\mu\left(\mathrm{A}_{\sigma \mathrm{i}-1)}\right)$. This kind of operation can be defined as a quasi-inverse of a $t-$ conorm operation $\hat{+}$ on $\mathrm{W}$, in the following way:

$$
\begin{aligned}
& \hat{\sim}: \mathrm{WxW} \rightarrow \mathrm{W} \\
& \mathrm{w}_{\mathrm{i}} \hat{\sim} \mathrm{w}_{\mathrm{j}}=\min \left\{\mathrm{w} \in \mathrm{W} / \mathrm{w} \hat{+} \mathrm{w}_{\mathrm{j}} \geq \mathrm{w}_{\mathrm{i}}\right\}
\end{aligned}
$$

for all $\mathrm{w}_{\mathrm{i}}, \mathrm{w}_{\mathrm{j}}$ in $\mathrm{W}$, where sup $\varnothing=0$. Notice that it always holds $\mathrm{w} \hat{\sim} \mathrm{w}=0$. Of course, if $\mathrm{W}=\{0,1,2$, ...., $\mathrm{n}\}$, the usual arithmetical addition $(+)$ and subtraction (-) in W correspond to take

$$
\mathrm{i} \hat{+} \mathrm{j}=\min (\mathrm{k}, \mathrm{i}+\mathrm{j})
$$

as $\mathrm{t}$-conorm in $\mathrm{W}$, and thus $\mathrm{i} \wedge \mathrm{j}=\max (0, \mathrm{ij})$, and $\hat{t}$ so defined is nothing but the Lukasiewicz $\mathrm{t}$ conorm on W. But this is only a particular case.

Once we have a pseudo-subtraction defined, next operation which is needed is a weighted mean-like operation of the criteria evaluations with the weights of the form $\mu\left(\mathrm{A}_{\sigma(\mathrm{i})}\right) \hat{=} \mu\left(\mathrm{A}_{\sigma(\mathrm{i}-1)}\right)$. A family of qualitative weighted mean operators have been recently introduced in [Godo and Torra, 1999] to average values from an ordinal scale $\mathrm{U}$ weighted with natural numbers. An associative, not bounded, addition-like operation $\Theta$ is defined from a tconorm operator $\oplus$ defined on (U, <), keeping track of accumulation. In more detail, we start out from a $\mathrm{t}$-conorm $\oplus: \mathrm{U} \times \mathrm{U} \rightarrow \mathrm{U}$ and we consider pairs of the type $(\mathrm{n}, \mathrm{x})$, where $\mathrm{n}$ is a natural number and $\mathrm{x} \in \mathrm{U}, \mathrm{x}$ $<1_{U}$. Then, an element $x<1_{U}$ is identified with the pair $(0, x)$, while $1_{U} \in U$ is identified with the pair $\left(1,0_{\mathrm{U}}\right)$. Over the set of pairs, an addition-like operation $\oplus$ is defined as follows:

$$
\begin{aligned}
& (\mathrm{n}, \mathrm{x}) \oplus(\mathrm{m}, \mathrm{y})= \\
& \begin{cases}(\mathrm{n}+\mathrm{m}, \mathrm{x} \oplus \mathrm{y}), & \text { if } \mathrm{x} \oplus \mathrm{y}<1_{\mathrm{U}} \\
(\mathrm{n}+\mathrm{m}+1, \mathrm{x} \otimes \mathrm{y}), & \text { if } \mathrm{x} \oplus \mathrm{y}=1_{\mathrm{U}}\end{cases}
\end{aligned}
$$

where $\otimes$ is the dual tnorm of $\oplus$, i.e. $u \otimes v=$ $\mathrm{n}_{\mathrm{U}}\left(\mathrm{n}_{\mathrm{U}}(\mathrm{u}) \oplus \mathrm{n}_{\mathrm{U}}(\mathrm{v})\right)$, $\mathrm{n}_{\mathrm{U}}$ being the natural involution in U. The simple and intuitive idea behind this definition is to view the elements of $U$ as the decimal part of generalized numbers, in such a way that if $u \oplus v=1_{U}$, then the integer part of the addition of $(n, u)$ with $(m, v)$ is $n+m$ augmented by 1 , and the decimal rest is estimated by the value $\mathrm{u} \otimes \mathrm{v}$. In this way, the bounded nature of the t-conorm is somehow disabled and it is possible to keep track of the implicit accumulation in $\oplus$. Of course, the degree of accumulation will depen on the chosen $t-$ conorm $\oplus$. For instance, if $\oplus=\max$, no accumulation will take place unless one of the two operands is $\mathrm{U}_{U}$, whereas if $\oplus=$ Lukasiewicz $^{1}$, the accumulation will be full.

The crucial point in this model is to make sure that the resulting addition operation be associative, otherwise the approach makes no sense. In [Godo and Torra, 1999] different sufficient conditions for the t-conorm $\oplus$ have been identified in order to generate an associative operation $\oplus$. One of the most relevant conditions is to be smooth, i.e. for any $\mathrm{u}$ and $\mathrm{v}$ of $\mathrm{U}$, if $\mathrm{u}<\mathrm{v}$ then there exists $\mathrm{w}$ such that $\mathrm{u}$ $\oplus \mathrm{w}=\mathrm{v}$. Smooth t-norms and t-conorms operations have been completely characterized (see for instance [Mayor and Torrens, 1988]) and it turns out that any smooth (finite) t-conorm is an ordinal sum of max and Lukasiewicz t-conorms, so they are easy to build up.

To our opinion, natural numbers correspond to the crudest view of a weight. Namely, if weights are to be considered only as elements of an ordinal scale, weights are nothing but their position in the scale, and thus they can be represented as indices (natural numbers). In such a case, for instance, thinking of a weighted average of a value $u$ weighted by 3 with a value $\mathrm{v}$ weighted by 5 , it is hard to imagine something else different from an average of 8 values, i.e. 3 repetitions of $u$ and 5 repetitions of $v$.

Therefore, according to this view, the weighting of a value $u$ by a natural $\mathrm{m}$, is defined as an iteration of the addition operation $\underline{\oplus}$, namely:

$$
\mathrm{m} \bullet \mathrm{u}=\mathrm{T}(\mathrm{u}) \underline{\oplus} . .{ }^{\mathrm{m})} . . \underline{\oplus} \mathrm{T}(\mathrm{u}),
$$

where $\mathrm{T}(\mathrm{u})=(0, \mathrm{u})$ if $\mathrm{u}<1_{\mathrm{U}}$, and $\mathrm{T}\left(1_{\mathrm{U}}\right)=\left(1,0_{\mathrm{U}}\right)$, which will result in another pair $(\mathrm{n}, \mathrm{x})$. Therefore, if the values to be aggregated are $\mathrm{v}_{1}, \ldots, \mathrm{v}_{\mathrm{k}} \in \mathrm{U}$, their Choquet-like integral with respect to a weighting measure $\mu$ on subsets of criteria corresponds to the expression:

1 By analogy to the numerical setting, the Lukasiewicz t-conorm $\oplus_{\mathrm{L}}$ on a finite scale $\mathrm{U}=\left\{\mathrm{u}_{0}\right.$ $\left.<\mathrm{u}_{1}<\ldots<\mathrm{u}_{\mathrm{r}}\right\}$ is defined by putting $\mathrm{u}_{\mathrm{i}} \oplus_{\mathrm{L}} \mathrm{u}_{\mathrm{i}}=\mathrm{u}_{\mathrm{h}}$, where $h=\min (r, i+j)$. 


$$
\begin{aligned}
& \left(\mu\left(\mathrm{A}_{\sigma(1)}\right)-\mu\left(\mathrm{A}_{\sigma(2)}\right)\right)^{\bullet} \mathrm{v}_{\sigma(1)} \oplus \ldots \\
& \quad \underline{\oplus}\left(\mu\left(\mathrm{A}_{\sigma(\mathrm{k})}\right)-\mu\left(\mathrm{A}_{\sigma(\mathrm{k}+1)}\right)\right) \boldsymbol{\bullet} \sigma(\mathrm{k})
\end{aligned}
$$

where $\sigma$ is a permutation of $\{1, \ldots, \mathrm{k}\}$ such that $\mathrm{v}_{\sigma(1)}$ $\leq v_{\sigma(2)} \leq \ldots \leq v_{\sigma(k)}$, and the sets $A_{\sigma(i)}$ 's are defined as above.

This is basically the approach taken in [Godo and Torra, 1999], where $\mathrm{W}$ is considered as the set of natural numbers \{ together with their usual arithmetic operations. However, as we have already mentioned before, the set of weights $\mathrm{W}$, as the domain of the discrete fuzzy measures on sets of criteria, needs to be bounded, and thus it would rather be a finite set of indices of the type $\{0,1, \ldots$, $\mathrm{n}\}$, where $\mathrm{n}$ is the measure of the total set $\mu(\mathrm{X})$. In this context, we can generalize the above approach by taking $\mathrm{W}$ also to build the extended values $(\mathrm{m}, \mathrm{u})$ together a t-conorm $\hat{+}: \mathrm{W} \times \mathrm{W} \rightarrow \mathrm{W}$ playing the role of addition. Notice that in this case, the t-conorm is indeed bounded but, as we shall see, this will not affect the construction.

$$
\begin{aligned}
& (\mathrm{n}, \mathrm{x}) \oplus(\mathrm{m}, \mathrm{y})= \\
& \begin{cases}(\mathrm{n} \hat{+} \mathrm{m}, \mathrm{x} \oplus \mathrm{y}), & \text { if } \mathrm{x} \oplus \mathrm{y}<1 \\
(\mathrm{n} \hat{+} \mathrm{m} \hat{+} 1, \mathrm{x} \otimes \mathrm{y}), & \text { if } \mathrm{x} \oplus \mathrm{y}=1\end{cases}
\end{aligned}
$$

Actually, it can be checked that if $\oplus$ generates an associative operation $\oplus$ in the sense of (1), it also generates an associative operation $\oplus$ in the sense of (3). In such a case, expression (2) becomes

$$
\begin{aligned}
& \left(\mu\left(\mathrm{A}_{\sigma(1)}\right) \wedge \mu\left(\mathrm{A}_{\sigma(2)}\right)\right) \bullet \mathrm{v}_{\sigma(1)} \stackrel{\oplus}{\ldots} .
\end{aligned}
$$

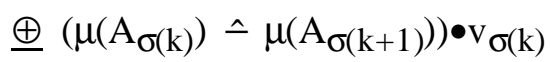

where we use the subtraction-like operation $\_$to compute the weights of the values

The result of the expressions (4) (or (2)) will be a pair $(\mathrm{m}, \mathrm{u})$, possibly with $\mathrm{m}>0$, but $\mathrm{m} \leq \mathrm{n}$. This is due to the fact that our qualitative fuzzy measure takes value on the set $\{0,1, . ., \mathrm{n}\}$, and in particular the measure of the total set of criteria $X$ is $n$. Thus, if we want the final result to be again a value of $U$, as it is usual in aggregation, we need to perform a kind of normalization. Following again [Godo and Torra, 1999], this can be done by using the quasi-inverse of the iteration operation $\bullet$. Namely, for any $\mathrm{m} \leq \mathrm{n}$ and $\mathrm{u}<1_{\mathrm{U}}$, we define

$$
(\mathrm{m}, \mathrm{u}) \varnothing \mathrm{n}=\max \left\{\mathrm{y} \in \mathrm{U} \mid \mathrm{n} \bullet \mathrm{y} \leq^{*}(\mathrm{~m}, \mathrm{u})\right\}
$$

where $\leq^{*}$ denotes the lexicographic ordering on pairs, that is, $(\mathrm{m}, \mathrm{u}) \mathrm{*}^{*}\left(\mathrm{~m}^{\prime}, \mathrm{u}^{\prime}\right)$ if either $\mathrm{m}<\mathrm{m}^{\prime}$ or $\mathrm{m}$ $=\mathrm{m}^{\prime}$ and $\mathrm{u}<\mathrm{u}$ '. Using this normalization, the final expression for the Choquet-like integral (type I) we propose for our framework is

$$
\begin{aligned}
(\mathrm{QC} 1) \int \mathrm{fd} \mu= & {\left[\left(\mu\left(\mathrm{A}_{\sigma(1)}\right) \hat{\sim} \mu\left(\mathrm{A}_{\sigma(2)}\right)\right) \bullet \mathrm{v}_{\sigma(1)} \oplus \ldots .\right.} \\
& \left.\oplus\left(\mu\left(\mathrm{A}_{\sigma(\mathrm{k})}\right) \hat{\sim} \mu\left(\mathrm{A}_{\sigma(\mathrm{k}+1)}\right)\right) \bullet \mathrm{v}_{\sigma(\mathrm{k})}\right] \varnothing \mathrm{n},
\end{aligned}
$$

where $f\left(x_{i}\right)=v_{i}$, for $i=1, k$. In the following we analyze the main properties of this discrete integral.

\section{Properties of the Qualitative Choquet integral of type I}

The first aspect to consider is the monotonicity of the Choquet integral with respect to the values to aggregate. This corresponds to study the monotonicity of $\oplus$. Since $\hat{+}, \oplus$ and $\otimes$ are indeed monotonic, non monotonicity can only arise in computations ( $\mathrm{p}, \mathrm{x}) \oplus(\mathrm{m}, \mathrm{y})$ when $\mathrm{x} \oplus \mathrm{y}=1$. This is the only problematic case. Regarding this problem we have the following easy results. We will write $\oplus=\mathrm{f}(\oplus, \hat{+})$ to make explicit that $\oplus$ is built from $\oplus$ and $\hat{+}$. We assume from now on that $\oplus$ is a smooth t-conorm on $\mathrm{U}$.

Proposition 1. Any smooth t-conorm $\oplus$ in $\mathrm{U}$ verifying $\mathrm{x} \oplus \mathrm{y}<1_{\mathrm{U}}$ for all $\mathrm{x}, \mathrm{y}<1_{\mathrm{U}}$ generates monotonic operators $\underline{\oplus}=\mathrm{f}(\oplus, \hat{+})$ for any $\hat{t}$.

This obvious result does not help very much since tconorms $\oplus$ verifying the condition of Proposition 1 do not allow for operators $\oplus$ which actually accumulate.

Proposition 2. Let $\hat{f}$ be a t-conorm in $\mathrm{W}$ for which there exists $\mathrm{m}<\mathrm{m}^{\prime} \in \mathrm{W}$ such that $\mathrm{p} \hat{+} \mathrm{m}=\mathrm{p} \hat{+} \mathrm{m}^{\prime}<\mathrm{n}$ for some $p \in W$. Then $\oplus=f(\oplus, \hat{+})$ does not satisfy monotonicity, for any $\oplus$.

Proof. Let $\hat{+}, \mathrm{m}, \mathrm{m}$ ' and $\mathrm{p}$ be as above. Further, consider $\mathrm{x}, \mathrm{y}$, y' of $\mathrm{U}$ such that $\mathrm{y}^{\prime}<\mathrm{y}$ and $\mathrm{x} \oplus \mathrm{y}^{\prime}<\mathrm{x}$ $\oplus \mathrm{y}<1_{\mathrm{U}}$. Such values can always be found since $\oplus$ is smooth. Then we have that $(\mathrm{p}, \mathrm{x}) \oplus(\mathrm{m}, \mathrm{y})=$ $(\mathrm{p} \hat{+} \mathrm{m}, \mathrm{x} \oplus \mathrm{y})>\left(\mathrm{p} \hat{+} \mathrm{m}^{\prime}, \mathrm{x} \oplus \mathrm{y}^{\prime}\right)=(\mathrm{p}, \mathrm{x}) \oplus\left(\mathrm{m}^{\prime}, \mathrm{y}^{\prime}\right)$ while it holds that $(\mathrm{m}, \mathrm{y})<\left(\mathrm{m}^{\prime}, \mathrm{y}^{\prime}\right)$. 
Corollary. The only smooth t-conorm $\hat{t}$ leading to a monotonic operator $\oplus=\mathrm{f}(\oplus, \hat{+})$ is the Lukasiewicz t-conorm.

Notice that if $\hat{+}$ is the Lukasiewicz tconorm, the fact that $\mathrm{p} \hat{+} \mathrm{m}=\mathrm{p} \hat{+} \mathrm{m}^{\prime}=\mathrm{n}$ causes no problem because when operating with $\oplus$, the maximum value that can ever be obtained is $\left(n, 0_{U}\right)$. This is so because, for any fuzzy measure $\mu$, the output (n, $\left.0_{U}\right)$ is obtained only with $v_{\sigma(i)}=\left(1,0_{U}\right)$ for all $\mathrm{i}$.

We consider now some other properties of this discrete integral.

Proposition 3. Let $\hat{+}$ be the Lukasiewicz t-conorm on $\mathrm{W}$. The qualitative Choquet integral of values $\mathrm{v}_{1}$, ..., $v_{k}$ of $U$ with respect to a qualitative $\hat{t}$ decomposable fuzzy measure $\mu$ on the set of criteria $\mathrm{C}=\left\{\mathrm{c}_{1}, \ldots, \mathrm{c}_{\mathrm{k}}\right\}$, that is, a measure such that $\mu(\mathrm{A} \cup \mathrm{B})$ $=\mu(\mathrm{A}) \hat{+} \mu(\mathrm{B})$ if $\mathrm{A} \cap \mathrm{B}=\varnothing$, reduces to the following expression:

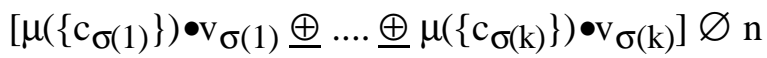

Thus it reduces to a qualitative weighted mean.

Proof. It is enough to consider a weighted mean with weights $\mathrm{w}_{\sigma(\mathrm{i})}=\left(\mu\left(\mathrm{A}_{\sigma(\mathrm{i})}\right) \hat{\sim} \mu\left(\mathrm{A}_{\sigma(\mathrm{i}+1)}\right)\right)$. Note that $\left(\mu\left(\mathrm{A}_{\sigma(\mathrm{i})}\right) \hat{-} \mu\left(\mathrm{A}_{\sigma(\mathrm{i}+1)}\right)\right)$ is just $\mu\left(\left\{\mathrm{c}_{\sigma(\mathrm{i})}\right\}\right)$ because $\mu$ is $\hat{t}$-decomposable, $\hat{t}$ being the Lukasiewicz $t-$ conorm, and thus, it holds that ( $\mathrm{w} \hat{+} \mathrm{a}) \hat{\sim} \mathrm{a}=\mathrm{w}$, for any $\mathrm{a}, \mathrm{w} \in \mathrm{W}$.

This proposition provides us with an analogous result to that for numerical Choquet integrals: a Choquet integral with an additive fuzzy measure is a weighted mean. Now, we formulate another result that is also the qualitative version of a well known result stating that numerical Choquet integrals with respect to a commutative fuzzy measure is indeed an OWA operator. By commutative qualitative fuzzy measure it is understood a measure $\mu$ such that $\mu(\mathrm{A})$ $=\mu(B)$ for all subsets B with the same cardinality as A.

Proposition 4. The qualitative Choquet integral of values $\mathrm{v}_{1}, \ldots, \mathrm{v}_{\mathrm{k}}$ of $\mathrm{U}$ with respect to a commutative fuzzy measure $\mu$ on the set of criteria $C=\left\{c_{1}, \ldots\right.$, $\left.c_{k}\right\}$ is of the form:

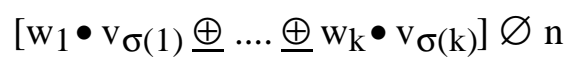

Thus becoming a Qualitative Ordered Weighting Aggregation (Q-OWA) operator in the sense of [Godo and Torra, 1999].

Proof. Notice that if $\mu$ is commutative we can safely define, for all $\mathrm{i} \in\{1, \ldots, \mathrm{k}\}, \mathrm{w}_{\mathrm{i}}=\mu(\mathrm{A}) \hat{\sim} \mu(\mathrm{B})$ for any sets $\mathrm{A}, \mathrm{B}$ such that $\mathrm{Card}(\mathrm{A})=\mathrm{i}$ and $\mathrm{Card}(\mathrm{B})=\mathrm{i}-$ 1. Then, the expression given above easily follows from our definition of type I qualitative Choquet integral of previous Section.

$\mathrm{M}$

Finally, let us relate the type I QCI to the qualitative counterpart of the so-called WOWA operators [Torra, 1997] introduced in [Godo and Torra, 1999]. Let $\mu$ be qualitative fuzzy measure on $\mathrm{X}$ over $\mathrm{W}$. We say that $\mu$ is a $\mathrm{Q}-\mathrm{w}$-decomposable fuzzy measure if, for any subset $\mathrm{A}$ of $\mathrm{X}, \mu(\mathrm{A})$ can be expressed as $\mu(\mathrm{A})=\mathrm{Q}\left(\hat{+}_{\mathrm{a} \in \mathrm{A}} \mathrm{w}(\mathrm{a})\right)$, where $\mathrm{w}: \mathrm{X} \rightarrow \mathrm{W}$ is a weighting vector and $\mathrm{Q}: \mathrm{W} \rightarrow \mathrm{W}$ is a nondecreasing quantifier, and $\hat{t}$ being the Lukasiewicz $\mathrm{t}$-conorm on $\mathrm{W}$.

Proposition 5. The qualitative Choquet integral of values $\mathrm{v}_{1}, \ldots, \mathrm{v}_{\mathrm{k}}$ of $\mathrm{U}$ with respect to a Q-pdecomposable fuzzy measure $\mu$ on the set of criteria $\mathrm{C}=\left\{\mathrm{c}_{1}, \ldots, \mathrm{c}_{\mathrm{k}}\right\}$ is of the form

$$
\begin{aligned}
& {\left[\left(\mathrm{q}_{1} \hat{\sim} \mathrm{q}_{2}\right) \bullet \mathrm{v}_{\sigma(1)} \oplus \ldots .\right.} \\
& \left.\quad \underline{\oplus}\left(\left[\left(\mathrm{q}_{\mathrm{k}} \hat{\sim} \mathrm{q}_{\mathrm{k}+1}\right)\right)\right) \bullet^{\bullet} \mathrm{v}_{\sigma(\mathrm{k})}\right] \varnothing \mathrm{n}
\end{aligned}
$$

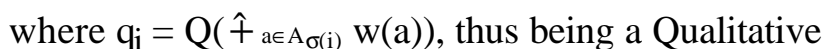
Weighted Ordered Weighted Averaging (QWOWA) operator.

When $\hat{t}$ is the Lukasiewicz t-conorm on $\mathrm{W}, \mathrm{Q}(\mathrm{x})=$ $\mathrm{x}$ for all $\mathrm{x} \in \mathrm{W}$, notice that this operator reduces to the qualitative weighted mean with weights $\mathrm{w}\left(\mathrm{x}_{\sigma(\mathrm{i})}\right)$ $=\mu\left(\left\{\mathrm{x}_{\sigma(\mathrm{i})}\right\}\right)$, and when $\mathrm{w}(\mathrm{a})=\alpha$ for all $\mathrm{a} \in \mathrm{X}$ reduces to a $\mathrm{Q}-\mathrm{OWA}$ with $\mathrm{w}_{\mathrm{i}}=\mathrm{Q}(\mathrm{i} \bullet \alpha) \wedge \mathrm{Q}((\mathrm{i}-1) \bullet \alpha)$.

\section{Qualitative Choquet Integral of type II}

Now what we want is to generalize the numerical Choquet integral expression

(C) $\int \mathrm{f} d \mu=\Sigma_{\mathrm{i}=1, \mathrm{k}}\left(\mathrm{f}\left(\mathrm{x}_{\sigma(\mathrm{i})}\right)-\mathrm{f}\left(\mathrm{x}_{\sigma(\mathrm{i}-1)}\right) \mu\left(\mathrm{A}_{\sigma(\mathrm{i})}\right)\right.$

to the qualitative setting. Based on the approach used in Section 3, we propose to rewrite (C2) in our 
qualitative framework as:

$$
\begin{aligned}
& (\mathrm{QC} 2) \int \mathrm{f} \mathrm{d} \mu= \\
& \qquad \underline{\oplus}_{\mathrm{i}=1, \mathrm{k}} \mu\left(\mathrm{A}_{\sigma(\mathrm{i})}\right) \bullet\left(\mathrm{f}\left(\mathrm{x}_{\sigma(\mathrm{i})}\right)--_{\oplus} \mathrm{f}\left(\mathrm{x}_{\sigma(\mathrm{i}-1)}\right)\right)
\end{aligned}
$$

where $\mathrm{f}: \mathrm{C} \rightarrow \mathrm{U}, \mu: 2^{\mathrm{C}} \rightarrow \mathrm{W}, \oplus$ is a smooth $\mathrm{t}$ conorm on $\mathrm{U}$, and $\oplus$ and $\bullet$ are defined as in the previous sections. We have introduced a subtractionlike $-_{\oplus}$ operator to compute the differences between values, instead of computing differences of measures as in Section 3. The subtraction operator $-\oplus$ is defined as the quasi-inverse operation of the $\mathrm{t}$ conorm $\oplus$. We study below two aspects of this integral. On the one hand its relation with fuzzy integrals and on the other hand its relationship to the Choquet integral of type I.

The so-called Fuzzy integral was introduced by Murofushi and Sugeno [Murofushi and Sugeno, 1991] to generalize in the unit interval the two main families of aggregation operators: (1) Choquet integrals and (2) Sugeno integrals. The former, as already mentioned, corresponds to other linear mean operators when selecting suitable fuzzy measures (e.g., the weighted mean or the OWA). The latter one corresponds to a weighted median (Prop 5.10 in [Fodor and Roubens, 1994]) generalizing some other median operators (e.g., the weighted minimum or the weighted maximum). We show here that the Qualitative Choquet integral introduced in the previous section can be also cast into the general notion of a fuzzy in tegral, provided some minor adaptations. We recall below the notion of Fuzzy integral, but we need some previous definitions.

Definition 1 [Murofushi and Sugeno, 1991]. $\mathrm{F}=(\Delta$, $\perp, \perp, \otimes)$ is a $t$-conorm system for integration iff

(a) $\Delta, \perp, \perp$ are continuous t-conorms, which are the maximum or Archimedean

(b) $\otimes:([0,1], \Delta) \times([0,1], \perp) \rightarrow([0,1], \perp)$ is a product-like operation fulfilling

(i) $\otimes$ is continuous on $] 0,1]^{2}$,

(ii) $\mathrm{a} \otimes \mathrm{x}=0 \Leftrightarrow \mathrm{a}=0$ or $\mathrm{x}=0$,

(iii) when $\mathrm{x} \perp \mathrm{y}<1, \mathrm{a} \otimes(\mathrm{x} \perp \mathrm{y})=$ $(\mathrm{a} \otimes \mathrm{x}) \perp(\mathrm{a} \otimes \mathrm{y})$, for all $\mathrm{a} \in[0,1]$,

(iv) when a $\Delta$ b $<1,(\mathrm{a} \Delta \mathrm{b}) \otimes \mathrm{x}=$ $(\mathrm{a} \otimes \mathrm{x}) \perp(\mathrm{b} \otimes \mathrm{x})$, for all $\mathrm{x} \in[0,1]$.
In this definition $([0,1], \Delta),([0,1], \perp),([0,1], \perp)$ correspond, respectively, to the spaces of values of integrand, measure and integral. According to the definition there are four types of t-systems:

(i) $\Delta, \perp$ and $\perp$ are Archimedean (Archimedean type t-system)

(ii) $\Delta=\perp=\perp=$ Maximum (maximum type t-system)

(iii) $\perp$ is Archimedean and at least one of the others is the maximum

(iv) $\perp=$ maximum and least one of the others is Archimedean

However, although these four types can be considered, only t-systems (i) and (ii) are meaningful when their properties are considered (see propositions 2.4 and 2.5 in [Murofushi and Sugeno, 1991] for details). One can already notice that $t$ systems (i) will generalize Choquet integrals while tsystems (ii) will generalize Sugeno integrals.

Definition 2 [Murofushi and Sugeno, 1991]. For a given t-conorm $\Delta$, we define an operation ${ }^{-} \Delta$ on $[0,1]^{2}$ by: $a-\Delta b:=\inf \{\mathrm{c} \mid \mathrm{b} \Delta \mathrm{c} \geq \mathrm{a}\}$.

Note that when $\Delta$ has a generator $\mathrm{k}$, then $\mathrm{a}-{ }_{\Delta} \mathrm{b}=$ $\mathrm{k}^{-1}[\max (0,(\mathrm{k}(\mathrm{a})-\mathrm{k}(\mathrm{b})))]$, and when $\Delta=$ maximum, we have $\mathrm{a}-{ }_{-} \mathrm{b}=\mathrm{a}$, if $\mathrm{a}>\mathrm{b} ; \mathrm{a}{ }_{-} \mathrm{b}=0$ otherwise.

Definition 3 [Murofushi and Sugeno, 1991]. Let (X, $X, \mu)$ be a fuzzy measure space and $(\Delta, \perp, \perp, \otimes)$ a t-system. Let $\mathrm{f}: \mathrm{X} \rightarrow[0,1]$ be a function such that $f(X)$ is finite. Let $f(X)=\left\{a_{0}, a_{1}, \ldots, a_{k}\right\}$ where $0=a_{0}$ $=\mathrm{a}_{1}=\mathrm{a}_{2}=\ldots=\mathrm{a}_{\mathrm{k}}=1$, and let $\mathrm{X}=\mathrm{A}_{0} \supseteq \mathrm{A}_{1} \supset \mathrm{A}_{2} \supset$ $\ldots \supset A_{k}$ be a family of sets with characteristic functions $\chi_{\mathrm{Ai}}$ such that $\mathrm{f}(\mathrm{x})=\max _{\mathrm{i}} \quad \mathrm{a}_{\mathrm{i}} \chi_{\mathrm{Ai}}(\mathrm{x})$, or equivalently, $\mathrm{f}(\mathrm{x})=\sum_{\mathrm{i}=1, \mathrm{k}}\left(\mathrm{a}_{\mathrm{i}}-\mathrm{a}_{\mathrm{i}-1}\right) \chi_{\text {Ai }}(\mathrm{x})$. The fuzzy $\mathrm{t}$-conorm integral (or fuzzy t-integral) of $\mathrm{f}$ based on $(\Delta, \perp, \perp, \otimes)$ with respect to $\mu$ is defined by the following expression:

$$
(\perp) \int \mathrm{f} \otimes \mathrm{d} \mu=\perp_{\mathrm{i}=1, \mathrm{k}}\left(\mathrm{a}_{\mathrm{i}}-{ }_{-} \mathrm{a}_{\mathrm{i}-1}\right) \otimes \mu\left(\mathrm{A}_{\mathrm{i}}\right)
$$

Our qualitative and discrete framework differs from the one of the fuzzy integral in the domains involved. In Definition 1, the integrand domain, the measure domain and the integral domain coincide, it is the unit interval $[0,1]$. However in our framework, the domains are different: the weight or measure domain is the set $\mathrm{W}$, the integrand domain is the ordinal scale $U$, and the integral domain is the cartesian product $\mathrm{Wx}(\mathrm{U}-\{1\})$. So, the fuzzy integral 
(5) corresponds in this case to (type II Choquet integral):

$\left(\underline{\oplus)} \int \mathrm{f} \otimes \mathrm{d} \mu=\bigoplus_{\mathrm{i}=1, \mathrm{k}} \mu\left(\mathrm{A}_{\sigma(\mathrm{i})}\right) \bullet\left(\mathrm{f}\left(\mathrm{x}_{\sigma(\mathrm{i})}\right)-_{\oplus} \mathrm{f}\left(\mathrm{x}_{\sigma(\mathrm{i}-1)}\right)\right)\right.$

Besides of that it can be proven that when the $\mathrm{t}$ conorm $\oplus$ used is Lukasiewicz this expression is equivalent to the type 1 Choquet integral.

Proposition 6. Qualitative Choquet integrals of type I (QC1) and of type II (QC2) are equal when the coresponding t-conorms $\oplus$ and $\hat{+}$ are Lukasiewicz.

\section{Examples}

Let us consider a qualitative version of the example described in [Grabisch, 1995]. The example consists on the evaluation of three students according to their level in three different subjects (criteria): Mathematics, Physics and Literature. The evaluation is computed with respect to a fuzzy measure $\mu: 2^{C}$ $\rightarrow \mathrm{W}$, where $\mathrm{C}=\{\mathrm{M}, \mathrm{P}, \mathrm{L}\}$ and $\mathrm{W}=\{0,1,2,3,4$, $5\}$, that satisfies the following assumptions (in the definition of the fuzzy measure subjects are represented by their corresponding first letter):

1) First we consider boundary conditions. The empty set has a null importance, and the set of all elements has a full importance.

$\mu(\varnothing)=0, \mu(\{\mathrm{M}, \mathrm{P}, \mathrm{L}\})=5$

(boundary conditions)

2) Relative importance of scientific versus literary subjects

$\mu(\{\mathrm{M}\})=\mu(\{\mathrm{P}\})=2, \mu(\{\mathrm{L}\})=1$ (singletons)

3) Redundancy between mathematics and physics

$\mu(\{\mathrm{M}, \mathrm{P}\})=3<2 \hat{+} 2=\mu(\{\mathrm{M}\}) \hat{+}(\{\mathrm{P}\})$ (measure on the pairs)

4) Support between literature and scientific subjects

$\mu(\{\mathrm{M}, \mathrm{L}\})=\mu(\{\mathrm{P}, \mathrm{L}\})=5>2 \hat{+} 1=$

$$
=\mu(\{\mathrm{M}\}) \quad \hat{+} \mu(\{\mathrm{L}\})=\mu(\{\mathrm{P}\}) \quad \hat{+} \quad(\{\mathrm{L}\})
$$

(measure on the pairs)

To develop this example we have used the valuations domain $U=(0,1,2,3,4,5)$ and using as the smooth t-conorm $\oplus$ given in Table 1 . In the set of weights $\mathrm{W}$ we shall use the Lukasiewicz $\mathrm{t}$-conorm $\hat{+}$.

\begin{tabular}{l||l|l|l|l|l|l|}
$\oplus$ & 0 & 1 & 2 & 3 & 4 & 5 \\
\hline \hline 0 & 0 & 1 & 2 & 3 & 4 & 5 \\
\hline 1 & 1 & 2 & 2 & 3 & 4 & 5 \\
\hline 2 & 2 & 2 & 2 & 3 & 4 & 5 \\
\hline 3 & 3 & 3 & 3 & 4 & 5 & 5 \\
\hline 4 & 4 & 4 & 4 & 5 & 5 & 5 \\
\hline 5 & 5 & 5 & 5 & 5 & 5 & 5 \\
\hline
\end{tabular}

Table 1. Smooth t-conorm on U used in the example

In Table 2 we display the level of the students and their evaluation using the qualitative weighted mean and both types of the qualitative Choquet integral. It can be seen that the results of the two types of Choquet integral are different as the t-conorm $\oplus$ used is not a Lukasiewicz tconorm. The Choquet integral of type I returns values that permit to order the students, while the other one does not allow this ordering as all students are evaluated with the same level. From now on, we consider only the results of the Choquet integral of type I.

$\begin{array}{lllllll}\text { Student } & \mathrm{M} & \mathrm{P} & \mathrm{L} & \mathrm{QWM} & \mathrm{QCI}-1 & \mathrm{QCI}-2 \\ \mathrm{~A} & 5 & 4 & 0 & 3 & 3 & 2 \\ \text { B } & 0 & 2 & 5 & 2 & 2 & 2 \\ \mathrm{C} & 4 & 4 & 4 & 3 & 4 & 2\end{array}$

Table 2. Qualitative evaluation of the students

To compare with the original example in [Grabisch, 1995] in Table 3 we display the numerical levels given by the author together with the computation using a weighted mean (with weights 3 for mathematics and physics and weight 2 for literature) and a Choquet integral (with a fuzzy measure defined as: $\mu(\{\})=0.0, \mu(\{\mathrm{M}\})=0.45, \mu(\{\mathrm{P}\})=0.45$, $\mu(\{\mathrm{L}\})=0.3, \mu(\{\mathrm{M}, \mathrm{L}\})=\mu(\{\mathrm{P}, \mathrm{L}\})=0.9$, $\mu(\{\mathrm{M}, \mathrm{P}\})=0.5, \mu(\{\mathrm{M}, \mathrm{P}, \mathrm{L}\})=1)$. 


\begin{tabular}{|c|c|c|c|c|c|}
\hline Student & M & $\mathrm{P}$ & $\mathrm{L}$ & WM & $\mathrm{CI}$ \\
\hline A & 18 & 16 & 10 & 15.25 & 13.9 \\
\hline B & 10 & 12 & 18 & 12.75 & 13.6 \\
\hline $\mathrm{C}$ & 14 & 15 & 15 & 14.62 & 14.9 \\
\hline
\end{tabular}

Table 3. Quantitative evaluation of the students

The results obtained are not equal but similar due in part to the fact that less precision is given. It can be observed that our qualitative assignment in Table 2 follows the linear order established in the quantitative assignment given in Table 3. I.e., the ordering $18>16>15>14>12>10$ is also satisfied in the qualitative case being $5>4 \geq 4 \geq 4$ $>2>0$. In the translation of numerical values to qualitative ones, the quantitative values of 16,15 and 14 are all translated into 4 as they are similar.

In relation to the final evaluation it should be said that the quantitative ordering is preserved in the qualitative setting for both the weighted mean and the Choquet integral. It should be noticed that the strict ordering in the numerical one is not strict in the qualitative setting. This is due to the fact that the qualitative case is less precise.

\section{Conclusions}

In this paper we have studied the extension of Choquet integrals to ordinal scales. We have presented two types of Choquet integrals showing that when the t-conorm $\oplus$ is Lukasiewicz both types collide. We have shown with an example the suitability of the approach to its application in real problems.

\section{Acknowledgments}

The authors acknowledge partial support of the CYCIT project SMASH (TIC96-1138-C04-01/04)

\section{References}

[1] Aczél, J., (1984), On weighted synthesis of judgements, Aequationes Math., 27 288-307.

[2] Choquet, G., (1968), Theory of Capacities, Ann. Inst. Fourier 5 (1968) 131-296.

[3] Dubois, D., Prade, H., (1986), A set-theoretic view of belief functions, Int. J. of General Systems 12 193-226.
[4] Fodor, J., Roubens, M., (1994), Fuzzy Preference Modelling and Multicriteria Decision Support, Kluwer Academic Publishers, Dordrecht, The Netherlands.

[5] Godo, L., Torra, V., (1999), On aggregation operators for ordinal qualitative information, IEEE Trans. on Fuzzy Systems (accepted).

[6] Grabisch, M., (1995), Fuzzy integral in multicriteria decision making, Fuzzy Sets and Systems 69 279-298.

[7] Grabisch, M., Nguyen, H. T., Walker, E. A., (1995), Fundamentals of Uncertainty Calculi with Applications to Fuzzy Inference, Kluwer Academic Publishers, Dordreht, The Netherlands.

[8] Herrera, F., Verdegay, J. L., (1993), Linguistic Assessments in group decision, Proc. of First European Congress on Fuzzy and Intelligent Technologies, Aachen, 941-948.

[9] Mayor, G. Torrens, J., (1993), On a Class of Operators for Expert Systems, Int. J. of Intelligent Systems, 8, 771-778.

[10] Murofushi, T., Sugeno, M., (1991), Fuzzy \& conorm integral with respect to fuzzy measures: generalization of Sugeno integral and Choquet integral, Fuzzy Sets and Systems, 42:1 57-71.

[11] Sugeno, M., (1974), Theory of Fuzzy Integrals and its Applications. (PhD Dissertation). Tokyo Institute of Technology, Tokyo, Japan.

[12] Torra, V., (1997), The Weighted OWA operator, Int. J. of Int. Systems, 12 (1997) 153166.

[13] Yager, R. R., (1988), On ordered weighted averaging aggregation operators in multi-criteria decision making, IEEE Trans. on SMC, 18 (1988) 183-190. 